\title{
Global Solutions to the Spherically Symmetric Compressible Navier-Stokes Equations with Density-Dependent Viscosity
}

\author{
Ruxu Lian, ${ }^{1}$ Lan Huang, ${ }^{1}$ and Jian Liu ${ }^{2}$ \\ ${ }^{1}$ College of Mathematics and Information Science, North China University of Water Resources and \\ Electric Power, Zhengzhou 450011, China \\ ${ }^{2}$ Department of Mathematics, Capital Normal University, Beijing 100048, China
}

Correspondence should be addressed to Ruxu Lian, ruxu.lian.math@gmail.com

Received 20 December 2011; Revised 21 March 2012; Accepted 31 March 2012

Academic Editor: Nazim I. Mahmudov

Copyright (c) 2012 Ruxu Lian et al. This is an open access article distributed under the Creative Commons Attribution License, which permits unrestricted use, distribution, and reproduction in any medium, provided the original work is properly cited.

We consider the exterior problem and the initial boundary value problem for the spherically symmetric isentropic compressible Navier-Stokes equations with density-dependent viscosity coefficient in this paper. For regular initial density, we show that there exists a unique global strong solution to the exterior problem or the initial boundary value problem, respectively. In particular, the strong solution tends to the equilibrium state as $t \rightarrow+\infty$.

\section{Introduction}

The isentropic compressible Navier-Stokes equations with density-dependent viscosity coefficients read as follows:

$$
\begin{gathered}
\rho_{t}+\operatorname{div}(\rho \mathbf{U})=0, \\
(\rho \mathbf{U})_{t}+\operatorname{div}(\rho \mathbf{U} \otimes \mathbf{U})+\nabla P(\rho)-\operatorname{div}(\mu(\rho) D(\mathbf{U}))-\nabla(\lambda(\rho) \operatorname{div} \mathbf{U})=0,
\end{gathered}
$$

where $t \in(0,+\infty)$ is the time and $\mathbf{x} \in R^{N}, N$ is the spatial coordinate, $\rho>0$ and $u$ denote the density and velocity, respectively. Pressure function is taken as $P(\rho)=\rho^{\gamma}$ with $\gamma>1$, and

$$
D(\mathbf{U})=\frac{\nabla(\mathbf{U})+{ }^{t} \nabla(\mathbf{U})}{2}
$$


is the strain tensor and $\mu(\rho), \lambda(\rho)$ are the Lamé viscosity coefficients satisfying

$$
\mu(\rho)>0, \quad \mu(\rho)+N \lambda(\rho) \geq 0
$$

There are many important progress achieved recently on the compressible NavierStokes equations with density-dependent viscosity coefficient. For instance, the mathematical derivations are derived in the simulation of flow surface in shallow region [1-4]. The prototype model is the viscous Saint-Venant. The well posedness of solutions to the free boundary value problem with initial finite mass and the flow density being connected with the infinite vacuum either continuously or via jump discontinuity is investigated by many authors, refer to [5-12] and references therein. Mellet and Vasseur showed the global existence of strong solutions for $\alpha \in(0,1 / 2)$ [13]. The qualitative behaviors of global solutions and dynamical asymptotics of vacuum states are also made, such as the finite time vanishing of finite vacuum or asymptotical formation of vacuum in large time, the dynamical behaviors of vacuum boundary, the large time convergence to rarefaction wave with vacuum, and the stability of shock profile with large shock strength, refer to [14-17] and references therein.

In this present paper, we consider the exterior problem and the initial boundary value problem for the spherically symmetric isentropic compressible Navier-Stokes equations with density-dependent viscosity coefficient and focus on the regularities and dynamical behaviors of global strong solution, and so forth. As $\gamma>1$, we show that the exterior problem and the initial boundary value problem with regular initial data both admit the unique global strong solution. In particular, the strong solution tends to the equilibrium state as $t \rightarrow+\infty$.

The rest of the paper is arranged as follows. In Section 2, the main results about the dynamical behaviors of global strong solution for compressible Navier-Stokes equations are stated. Then, the theorems of the exterior problem and the initial boundary value problem are proved in Sections 3 and 4, respectively.

\section{Notations and Main Results}

For simplicity, we will take $\mu(\rho)=\rho$ and $\lambda(\rho)=0$ and $D(\mathbf{U})=\nabla \mathbf{U}$ in (1.1). The isentropic compressible Navier-Stokes equations become

$$
\begin{gathered}
\rho_{t}+\operatorname{div}(\rho \mathbf{U})=0 \\
(\rho \mathbf{U})_{t}+\operatorname{div}(\rho \mathbf{U} \otimes \mathbf{U})+\nabla P(\rho)-\operatorname{div}(\rho \nabla \mathbf{U})=0 .
\end{gathered}
$$

Firstly, we consider the exterior problem, namely, the initial data and boundary conditions of (2.1) are imposed as follows:

$$
\begin{gathered}
(\rho, \mathbf{U})(\mathbf{x}, 0)=\left(\rho_{0}, \mathbf{U}_{0}\right)(\mathbf{x}), \quad \mathbf{x} \in \Omega, \\
\mathbf{U}=0, \quad \text { on } \partial \Omega, \quad \lim _{|\mathbf{x}| \rightarrow+\infty}(\rho, u)(\mathbf{x}, t)=(\bar{\rho}, 0), \quad t \in[0, T],
\end{gathered}
$$

where $\Omega:=R^{3} / \Omega_{r_{-}}, \Omega_{r_{-}}$is a ball of radius $r_{-}$centered at the origin in $R^{3}$, and $\bar{\rho}>0$ is a constant. 
We are concerned with the spherically symmetric solutions of system (2.1) in an spherically symmetric exterior domain $\Omega$. To this end, we denote that

$$
|\mathbf{x}|=r, \quad \rho(\mathbf{x}, t)=\rho(r, t), \quad \mathbf{U}(\mathbf{x}, t)=u(r, t) \frac{\mathbf{x}}{r},
$$

which leads to the following system of equations for $r>0$,

$$
\begin{gathered}
\rho_{t}+(\rho u)_{r}+\frac{2 \rho u}{r}=0 \\
(\rho u)_{t}+\left(\rho u^{2}+\rho^{r}\right)_{r}+\frac{2 \rho u^{2}}{r}-\left(\rho u_{r}\right)_{r}-\rho\left(\frac{2 u}{r}\right)_{r}=0
\end{gathered}
$$

with the initial data and boundary conditions

$$
\begin{gathered}
(\rho, u)(r, 0)=\left(\rho_{0}, u_{0}\right)(r), \quad r \in\left[r_{-},+\infty\right), \\
u\left(r_{-}, t\right)=0, \quad \lim _{r \rightarrow+\infty}(\rho, u)(r, t)=(\bar{\rho}, 0) \quad t \in[0, T],
\end{gathered}
$$

and the initial data satisfies for some constants $\bar{\rho}$ and $\underline{\rho}>0$

$$
\begin{gathered}
\rho_{0}-\bar{\rho} \in L^{1}\left(\left[r_{-},+\infty\right)\right) \cap L^{2}\left(\left[r_{-},+\infty\right)\right), \quad \inf _{\mathbf{x} \in\left[r_{-}+\infty\right)} \rho_{0}>\underline{\rho}>0, \\
r \rho_{0 r} \in L^{2}\left(\left[r_{-},+\infty\right)\right), \quad r^{2} u_{0} \in H^{2}\left(\left[r_{-},+\infty\right)\right) .
\end{gathered}
$$

Then, we define that

$$
\begin{aligned}
& E_{0}:=\frac{1}{2} \int_{r_{-}}^{+\infty} \rho_{0} u_{0}^{2} r^{2} d r+\int_{r_{-}}^{+\infty} \rho_{0}\left(\frac{1}{r-1}\left(\rho_{0}^{\gamma-1}-\bar{\rho}^{\gamma-1}\right)+\bar{\rho}^{\gamma}\left(\rho_{0}^{-1}-\bar{\rho}^{-1}\right)\right) r^{2} d r, \\
& E_{1}:=\frac{1}{2} \int_{r_{-}}^{+\infty} \rho_{0}\left(u_{0}+\rho_{0}^{-1}\left(\rho_{0}\right)_{r}\right)^{2} r^{2} d r+\int_{r_{-}}^{+\infty} \rho_{0}\left(\frac{1}{r-1}\left(\rho_{0}^{\gamma-1}-\bar{\rho}^{\gamma-1}\right)+\bar{\rho}^{\gamma}\left(\rho_{0}^{-1}-\bar{\rho}^{-1}\right)\right) r^{2} d r,
\end{aligned}
$$

and give the main results as follows.

Theorem 2.1. Let $\gamma>1$. Assume that the initial data satisfies (2.6) and $E_{0}+E_{1}<v r_{-}^{2} \bar{\rho}^{\gamma / 2+1 / 2}, v$ is a positive constant. Then, there exist two positive constants $\rho_{*}$ and $\rho^{*}$ and a unique global strong solution $(\rho, u)$ to the exterior problem (2.4) and (2.5), namely, satisfying

$$
\begin{gathered}
0<\rho_{*} \leq \rho(r, t) \leq \rho^{*}, \quad(r, t) \in\left[r_{-},+\infty\right) \times[0, T], \\
\rho-\bar{\rho} \in L^{\infty}\left([0, T] ; L^{2}\left(\left[r_{-},+\infty\right)\right)\right), \quad \rho_{r} \in L^{\infty}\left([0, T] ; L^{2}\left(\left[r_{-},+\infty\right)\right)\right), \\
u \in L^{\infty}\left([0, T] ; L^{2}\left(\left[r_{-},+\infty\right)\right)\right), \quad u_{r} \in L^{\infty}\left([0, T] ; L^{2}\left(\left[r_{-},+\infty\right)\right)\right) .
\end{gathered}
$$

Furthermore, the solution tends to the equilibrium state $(\bar{\rho}, 0)$ 


$$
\|(\rho-\bar{\rho}, u)(\cdot, t)\|_{L^{\infty}\left(\left[r_{-},+\infty\right)\right)} \longrightarrow 0, \quad t \longrightarrow+\infty .
$$

Then investigates the initial boundary value problem, and the initial data and boundary conditions of (2.1) are assumed as follow:

$$
\begin{gathered}
(\rho, \mathbf{U})(\mathbf{x}, 0)=\left(\rho_{0}, \mathbf{U}_{0}\right)(\mathbf{x}), \quad \mathbf{x} \in \Omega, \\
\mathbf{U}(\mathbf{x}, t)=0, \quad \mathbf{x} \in \partial \Omega, t \in[0, T] .
\end{gathered}
$$

By (2.3), one considers (2.4) with the initial data and boundary conditions

$$
\begin{gathered}
(\rho, u)(r, 0)=\left(\rho_{0}, u_{0}\right)(r), \quad r \in\left[r_{-}, r_{+}\right] \\
u\left(r_{-}, t\right)=u\left(r_{+}, t\right)=0, \quad t \in[0, T]
\end{gathered}
$$

and the initial data satisfies for some constant $\underline{\rho}>0$

$$
\begin{gathered}
\rho_{0} \in L^{1}\left(\left[r_{-}, r_{+}\right]\right) \cap W^{1, \infty}\left(\left[r_{-}, r_{+}\right]\right), \inf _{x \in\left[r_{-}, r_{+}\right]} \rho_{0}>\underline{\rho}>0, \\
r^{2} u_{0} \in H^{2}\left(\left[r_{-}, r_{+}\right]\right) .
\end{gathered}
$$

Then, can give the main results as follows.

Theorem 2.2. Let $\gamma>1$. Assume that the initial data satisfies (2.12), there exist two positive constants $\rho_{*}$ and $\rho^{*}$ and a unique global strong solution $(\rho, u)$ to the initial boundary value problem (2.4) and (2.11), namely, satisfying

$$
\begin{array}{cl}
0<\rho_{*} \leq \rho(r, t) \leq \rho^{*}, & (r, t) \in\left[r_{-}, r_{+}\right] \times[0, T], \\
\rho \in L^{\infty}\left([0, T] ; L^{2}\left(\left[r_{-}, r_{+}\right]\right)\right), & \rho_{r} \in L^{\infty}\left([0, T] ; L^{2}\left(\left[r_{-}, r_{+}\right]\right)\right), \\
u \in L^{\infty}\left([0, T] ; L^{2}\left(\left[r_{-}, r_{+}\right]\right)\right), & u_{r} \in L^{\infty}\left([0, T] ; L^{2}\left(\left[r_{-}, r_{+}\right]\right)\right) .
\end{array}
$$

Furthermore, the solution $(\rho, u)$ tends to the equilibrium state exponentially

$$
\|(\rho-\tilde{\rho}, u)(\cdot, t)\|_{L^{\infty}\left(\left[r_{-}, r_{+}\right]\right)} \leq C_{0} e^{-C_{1} t}
$$

where $C_{0}$ and $C_{1}$ are positive constants independent of time and $\tilde{\rho}=\int_{r_{-}}^{r_{+}} \rho(r, t) r^{2} d r$. 
Remark 2.3. Theorems 2.1 and 2.2 hold for one-dimensional Saint-Venant's model for shallow water, that is, $\gamma=2, \alpha=1$.

\section{Proof of the Exterior Problem}

\subsection{The A Priori Estimates}

It is convenient to make use of the Lagrangian coordinates so as to establish the uniformly a priori estimates. Take the Lagrange coordinates transform

$$
x=\int_{r_{-}}^{r} \rho(r, t) r^{2} d r, \quad \tau=t
$$

which map $(r, t) \in\left[r_{-},+\infty\right) \times R^{+}$into $(x, \tau) \in[0,+\infty) \times R^{+}$. The relation between Lagrangian and Eulerian coordinates are satisfied as

$$
\frac{\partial x}{\partial r}=\rho r^{2}, \quad \frac{\partial x}{\partial t}=-\rho u r^{2}
$$

The exterior problem (2.4) and (2.5) is reformulated to

$$
\begin{gathered}
\rho_{\tau}+\rho^{2}\left(r^{2} u\right)_{x}=0, \\
r^{-2} u_{\tau}+\left(\rho^{r}\right)_{x}=\left(\rho^{2}\left(r^{2} u\right)_{x}\right)_{x}-\frac{2 \rho_{x} u}{r}, \\
(\rho, u)(x, 0)=\left(\rho_{0}, u_{0}\right)(x), \quad x \in[0,+\infty), \\
u(0, t)=0, \quad \lim _{x \rightarrow+\infty}(\rho, u)=(\bar{\rho}, 0), \quad \tau \in[0,+\infty),
\end{gathered}
$$

where the initial data satisfies

$$
\begin{gathered}
\rho_{0}-\bar{\rho} \in L^{1}([0,+\infty)) \cap L^{2}([0,+\infty)), \quad \inf _{x \in[0,+\infty)} \rho_{0}>\rho_{-}>0, \\
r^{2} \rho_{0 x} \in L^{2}([0,+\infty)), \quad \frac{1}{\sqrt{r^{2} \rho_{0}}}\left(r^{2} u_{0}\right) \in L^{2}([0,+\infty)), \\
\sqrt{r^{2} \rho_{0}}\left(r^{2} u_{0}\right)_{x} \in L^{2}([0,+\infty)), \quad \frac{1}{\sqrt{r^{2} \rho_{0}}}\left(r^{2} \rho\left(r^{2} \rho\left(r^{2} u_{0}\right)_{x}\right)_{x}\right) \in L^{2}([0,+\infty)) .
\end{gathered}
$$

First, we will establish the a-priori estimates for the solution $(\rho, u)$ to the exterior problem (3.3). 
Lemma 3.1. Let $T>0$. Under the conditions in Theorem 2.1, it holds for any solution $(\rho, u)$ to the exterior problem (3.3) that

$$
\begin{gathered}
\frac{1}{2} \int_{0}^{+\infty} u^{2} d x+\int_{0}^{+\infty}\left(\frac{1}{r-1}\left(\rho^{\gamma-1}-\bar{\rho}^{\gamma-1}\right)+\bar{\rho}^{\gamma}\left(\rho^{-1}-\bar{\rho}^{-1}\right)\right) d x \\
+\int_{0}^{\tau} \int_{0}^{+\infty}\left(\frac{2 u^{2}}{r^{2}}+\rho^{2} u_{x}^{2} r^{4}\right) d x d s \leq E_{0}, \quad \tau \in[0, T]
\end{gathered}
$$

Proof. Multiplying $(3.3)_{2}$ by $r^{2} u$ and integrating the result with respect to $x$ over $[0,+\infty)$, making use of (3.3) $)_{1}$ and (3.4), we have

$$
\begin{aligned}
& \frac{1}{2} \frac{d}{d \tau} \int_{0}^{+\infty} u^{2} d x+\frac{d}{d \tau} \int_{0}^{+\infty}\left(\frac{1}{\gamma-1}\left(\rho^{\gamma-1}-\bar{\rho}^{\gamma-1}\right)+\bar{\rho}^{\gamma}\left(\rho^{-1}-\bar{\rho}^{-1}\right)\right) d x+\int_{0}^{+\infty} \rho^{2}\left(r^{2} u\right)_{x}^{2} d x \\
& \quad=2 \int_{0}^{+\infty} \rho\left(r u^{2}\right)_{x} d x
\end{aligned}
$$

integrating (3.6) with respect to $\tau$, we obtain

$$
\begin{gathered}
\frac{1}{2} \int_{0}^{+\infty} u^{2} d x+\int_{0}^{+\infty}\left(\frac{1}{r-1}\left(\rho^{\gamma-1}-\bar{\rho}^{\gamma-1}\right)+\bar{\rho}^{\gamma}\left(\rho^{-1}-\bar{\rho}^{-1}\right)\right) d x+\int_{0}^{\tau} \int_{0}^{+\infty}\left(\frac{2 u^{2}}{r^{2}}+\rho^{2} u_{x}^{2} r^{4}\right) d x d s \\
=\frac{1}{2} \int_{0}^{+\infty} u_{0}^{2} d x+\int_{0}^{+\infty}\left(\frac{1}{\gamma-1}\left(\rho_{0}^{\gamma-1}-\bar{\rho}^{\gamma-1}\right)+\bar{\rho}^{\gamma}\left(\rho_{0}^{-1}-\bar{\rho}^{-1}\right)\right) d x
\end{gathered}
$$

Lemma 3.1 can be obtained.

Lemma 3.2. Let $T>0$. Under the conditions in Theorem 2.1, it holds for any solution $(\rho, u)$ to the exterior problem (3.3) that

$$
\begin{aligned}
& \frac{1}{2} \int_{0}^{+\infty}\left(u+r^{2} \rho_{x}\right)^{2} d x+\int_{0}^{+\infty}\left(\frac{1}{\gamma-1}\left(\rho^{\gamma-1}-\bar{\rho}^{\gamma-1}\right)+\bar{\rho}^{\gamma}\left(\rho^{-1}-\bar{\rho}^{-1}\right)\right) d x \\
& \quad+\gamma \int_{0}^{\tau} \int_{0}^{+\infty} \rho^{\gamma-1} \rho_{x}^{2} r^{4} d x d s \leq E_{1}, \quad \tau \in[0, T]
\end{aligned}
$$

and there exist two constants $0<\rho_{*}<\rho^{*}$ such that

$$
0<\rho_{*} \leq \rho(x, \tau) \leq \rho^{*}, \quad(x, \tau) \in[0,+\infty) \times[0, T] .
$$

Proof. Differentiating (3.3) ${ }_{1}$ with respect to $x$, we have

$$
\rho_{x \tau}+\left(\rho^{2}\left(r^{2} u\right)_{x}\right)_{x}=0 .
$$


Journal of Applied Mathematics

Summing (3.10) and (3.3) 2 , we get

$$
\left(r^{-2} u+\rho_{x}\right)_{\tau}+\left(\rho^{r}\right)_{x}=\left(r^{-2}\right)_{\tau} u-\frac{2 \rho_{x} u}{r} .
$$

Note that

$$
r^{3}(x, \tau)=r_{-}^{3}+3 \int_{0}^{x} \frac{1}{\rho(z, \tau)} d z
$$

and so

$$
\frac{\partial r}{\partial \tau}=\frac{1}{r^{2}} \int_{0}^{x}\left(\frac{1}{\rho}\right)_{t}(z, t) d z=\frac{1}{r^{2}} \int_{0}^{x}\left(r^{2} u\right)_{z}(z, t) d z=u(x, \tau),
$$

which together with (3.11) yields

$$
\left(r^{-2} u+\rho_{x}\right)_{\tau}+\left(\rho^{r}\right)_{x}=-2 r^{-3} u^{2}-\frac{2 \rho_{x} u}{r} .
$$

Multiplying (3.14) by $\left(u+r^{2} \rho_{x}\right) r^{2}$ and integrating the result with respect to $x$ and $\tau$, we have

$$
\begin{aligned}
& \frac{1}{2} \int_{0}^{+\infty}\left(u+r^{2} \rho_{x}\right)^{2} d x+\int_{0}^{+\infty}\left(\frac{1}{\gamma-1}\left(\rho^{\gamma-1}-\bar{\rho}^{\gamma-1}\right)+\bar{\rho}^{\gamma}\left(\rho^{-1}-\bar{\rho}^{-1}\right)\right) d x \\
& \quad+\gamma \int_{0}^{\tau} \int_{0}^{+\infty} \rho^{\gamma-1} \rho_{x}^{2} r^{4} d x d s \\
& \quad=\frac{1}{2} \int_{0}^{+\infty}\left(u_{0}+r^{2} \rho_{0 x}\right)^{2} d x+\int_{0}^{+\infty}\left(\frac{1}{\gamma-1}\left(\rho_{0}^{\gamma-1}-\bar{\rho}^{\gamma-1}\right)+\bar{\rho}^{\gamma}\left(\rho_{0}^{-1}-\bar{\rho}^{-1}\right)\right) d x .
\end{aligned}
$$

Let

$$
\begin{gathered}
\varphi(\rho):=\frac{1}{\gamma-1}\left(\rho^{\gamma-1}-\bar{\rho}^{\gamma-1}\right)+\bar{\rho}^{\gamma}\left(\rho^{-1}-\bar{\rho}^{-1}\right), \\
\psi(\rho):=\int_{\bar{\rho}}^{\rho} \varphi(\eta)^{1 / 2} d \eta .
\end{gathered}
$$

It follows from (3.6) and (3.13) that

$$
\begin{aligned}
|\psi(\rho)| & \leq\left|\int_{y}^{+\infty} \partial_{x} \psi(\rho) d x\right| \leq r_{-}^{-2}\left|\int_{y}^{+\infty} \varphi(\rho)^{1 / 2} \rho_{x} r^{2} d x\right| \\
& \leq r_{-}^{-2}\left|\int_{0}^{+\infty} \varphi(\rho) d y \int_{0}^{+\infty}\left(r^{2} \rho_{x}\right)^{2} d x\right|^{1 / 2} \leq r_{-}^{-2}\left(E_{0}+E_{1}\right) .
\end{aligned}
$$

We can verify that 
(1) As $\rho \rightarrow+\infty$, it holds for $\xi=\theta \bar{\rho}+(1-\theta) \rho$, where $\theta \in(0,1)$

$$
\begin{aligned}
\lim _{\rho \rightarrow+\infty} \psi(\rho) & =\lim _{\rho \rightarrow+\infty} \int_{\bar{\rho}}^{\rho}\left((\gamma-2) \xi^{\gamma-3}+2 \bar{\rho}^{\gamma} \xi^{-3}\right)^{1 / 2}|\eta-\bar{\rho}| d \eta \\
& \geq \lim _{\rho \rightarrow+\infty}\left((\gamma-2) \xi^{\gamma-3}+2 \bar{\rho}^{\gamma} \xi^{-3}\right)^{1 / 2} \int_{\bar{\rho}}^{\rho}(\eta-\bar{\rho}) d \eta \\
& =\lim _{\rho \rightarrow+\infty} \frac{1}{2}\left((\gamma-2)(\theta \bar{\rho}+(1-\theta) \rho)^{\gamma-3}+2 \bar{\rho}^{\gamma}(\theta \bar{\rho}+(1-\theta) \rho)^{-3}\right)^{1 / 2}(\rho-\bar{\rho})^{2} \\
& \longrightarrow+\infty .
\end{aligned}
$$

(2) As $\rho \rightarrow 0$, it holds for $\xi=\theta \bar{\rho}+(1-\theta) \rho$, where $\theta \in(0,1)$

$$
\begin{aligned}
\lim _{\rho \rightarrow 0} \psi(\rho) & =\lim _{\rho \rightarrow 0} \int_{\bar{\rho}}^{\rho}\left((\gamma-2) \xi^{\gamma-3}+2 \bar{\rho}^{\gamma} \xi^{-3}\right)^{1 / 2}|\eta-\bar{\rho}| d \eta \\
& \leq-\lim _{\rho \rightarrow 0}\left((\gamma-2) \xi^{\gamma-3}+2 \bar{\rho}^{\gamma} \xi^{-3}\right)^{1 / 2} \int_{\bar{\rho}}^{\rho}(\eta-\bar{\rho}) d \eta \\
& =-\lim _{\rho \rightarrow 0} \frac{1}{2}\left((\gamma-2)(\theta \bar{\rho}+(1-\theta) \rho)^{\gamma-3}+2 \bar{\rho}^{\gamma}(\theta \bar{\rho}+(1-\theta) \rho)^{-3}\right)^{1 / 2}(\rho-\bar{\rho})^{2} \\
& =:-v \bar{\rho}^{\gamma / 2+1 / 2}
\end{aligned}
$$

Applying (3.17)-(3.19) and $E_{0}+E_{1}<v r_{-}^{2} \bar{\rho}^{\gamma / 2+1 / 2}$, where $v$ is a positive constant, we can prove (3.9).

Lemma 3.3. Let $T>0$. Under the conditions in Theorem 2.1, it holds for any solution $(\rho, u)$ to the exterior problem (3.3) that

$$
\begin{aligned}
\int_{0}^{+\infty} & \left(r^{2} u\right)_{x}^{2} d x+\int_{0}^{+\infty}\left(r^{2} u\right)_{\tau}^{2} r^{-4} d x+\int_{0}^{\tau} \int_{0}^{+\infty}\left(r^{2} u\right)_{s}^{2} r^{-4} d x d s \\
& +\int_{0}^{\tau} \int_{0}^{+\infty} \rho^{2}\left(r^{2} u\right)_{x s}^{2} d x d s+\int_{0}^{\tau} \int_{0}^{+\infty}\left(r^{2} u\right)_{x x}^{2} d x d s \leq C, \quad \tau \in[0, T]
\end{aligned}
$$

where $C>0$ denotes a constant independent of time. 
Proof. Multiplying $(3.3)_{2}$ by $\rho^{-2}\left(r^{2} u\right)_{\tau}$ and integrating the result with respect to $x$ over $[0,+\infty)$, making use of $(3.4)$, we obtain

$$
\begin{aligned}
& \frac{d}{d \tau} \int_{0}^{+\infty}\left(\frac{1}{2}\left(r^{2} u\right)_{x}^{2}-\rho^{r-2}\left(r^{2} u\right)_{x}\right) d x+\int_{0}^{+\infty} \rho^{-2}\left(r^{2} u\right)_{\tau}^{2} r^{-4} d x \\
& =(r-2) \int_{0}^{+\infty} \rho^{\gamma-1}\left(r^{2} u\right)_{x}^{2} d x-2 \int_{0}^{+\infty} \rho^{\gamma-3} \rho_{x}\left(r^{2} u\right)_{\tau} d x+2 \int_{0}^{+\infty} \rho^{-1} \rho_{x}\left(r^{2} u\right)_{x}\left(r^{2} u\right)_{\tau} d x \\
& \quad+2 \int_{0}^{+\infty} \rho^{-2} u^{2}\left(r^{2} u\right)_{\tau} r^{-3} d x-2 \int_{0}^{+\infty} \rho^{-2} \rho_{x} u\left(r^{2} u\right)_{\tau} r^{-1} d x
\end{aligned}
$$

which implies

$$
\begin{aligned}
& \int_{0}^{+\infty}\left(r^{2} u\right)_{x}^{2} d x+\int_{0}^{\tau} \int_{0}^{+\infty}\left(r^{2} u\right)_{s}^{2} r^{-4} d x d s \\
& \leq C+C \int_{0}^{+\infty}\left(\rho^{r-2}-\bar{\rho}^{\gamma-2}\right)^{2} d x+C \int_{0}^{\tau} \int_{0}^{+\infty}\left(\frac{u^{2}}{r^{2}}+u_{x}^{2} r^{4}\right) d x d s+C \int_{0}^{\tau} \int_{0}^{+\infty} \rho_{x}^{2} r^{4} d x d s \\
& \quad+C \int_{0}^{\tau} \int_{0}^{+\infty} \rho_{x}^{2}\left(r^{2} u\right)_{x}^{2} r^{4} d x d s+C \int_{0}^{\tau} \int_{0}^{+\infty} u^{4} r^{-2} d x d s+C \int_{0}^{\tau} \int_{0}^{+\infty} \rho_{x}^{2} u^{2} r^{2} d x d s \\
& \leq C+C \int_{0}^{\tau} \int_{0}^{+\infty} \rho_{x}^{2}\left(r^{2} u\right)_{x}^{2} r^{4} d x d s+C \sup _{\tau \in[0, T]}\|u\|_{L^{\infty}}^{2} .
\end{aligned}
$$

From (3.3) 2 , (3.5), (3.8), and (3.9), we can deduce that for some small $\epsilon \in(0,1)$

$$
\begin{aligned}
& \int_{0}^{\tau} \int_{0}^{+\infty} \rho_{x}^{2}\left(r^{2} u\right)_{x}^{2} r^{4} d x d s \leq \epsilon \int_{0}^{\tau} \int_{0}^{+\infty} \rho_{x}^{2}\left(r^{2} u\right)_{x}^{2} r^{4} d x d s+\epsilon \int_{0}^{\tau} \int_{0}^{+\infty}\left(r^{2} u\right)_{x s}^{2} r^{-4} d x d s \\
&+\epsilon \int_{0}^{\tau} \int_{0}^{+\infty} \rho_{x}^{2} d x d s+C(\epsilon) \int_{0}^{\tau} \int_{0}^{+\infty}\left(\frac{u^{2}}{r^{2}}+u_{x}^{2} r^{4}\right) d x d s, \\
& \sup _{\tau \in[0, T]}\|u\|_{L^{\infty}}^{2} \leq \epsilon \sup _{\tau \in[0, T]} \int_{0}^{+\infty}\left(r^{2} u\right)_{x}^{2} d x+C(\epsilon) \sup _{\tau \in[0, T]} \int_{0}^{+\infty} u^{2} d x,
\end{aligned}
$$

using (3.22)-(3.24), we can obtain that

$$
\int_{0}^{+\infty}\left(r^{2} u\right)_{x}^{2} d x+\int_{0}^{\tau} \int_{0}^{+\infty}\left(r^{2} u\right)_{s}^{2} r^{-4} d x d s \leq C+C \epsilon \int_{0}^{\tau} \int_{0}^{+\infty}\left(r^{2} u\right)_{x s}^{2} r^{-4} d x d s
$$


Differentiating (3.3) $)_{2}$ with respect to $\tau$, multiplying the result by $\left(r^{2} u\right)_{\tau}$, and integrating the result with respect to $x$ over $[0,+\infty)$, we have

$$
\begin{aligned}
\frac{1}{2} \frac{d}{d \tau} & \int_{0}^{+\infty}\left(r^{2} u\right)_{\tau}^{2} r^{-4} d x+\int_{0}^{+\infty} \rho^{2}\left(r^{2} u\right)_{x \tau}^{2} d x \\
= & 2 \int_{0}^{+\infty} u u_{\tau}\left(r^{2} u\right)_{\tau} r^{-3} d x-\frac{1}{2} \int_{0}^{+\infty}\left(r^{-4}\right)_{\tau}\left(r^{2} u\right)_{\tau}^{2} d x+2 \int_{0}^{+\infty} u\left(r^{-1} u\right)_{\tau}\left(r^{2} u\right)_{\tau} r^{-2} d x \\
& +\int_{0}^{+\infty}\left(\rho^{r}\right)_{\tau}\left(r^{2} u\right)_{x \tau} d x-\int_{0}^{+\infty}\left(\rho^{2}\right)_{\tau}\left(r^{2} u\right)_{x}\left(r^{2} u\right)_{x \tau} d x-\int_{0}^{+\infty}\left(\frac{2 \rho_{x} u}{r}\right)_{\tau}\left(r^{2} u\right)_{\tau} d x \\
& +2 \int_{0}^{+\infty}\left(r^{-2}\right)_{\tau} r^{-1} u^{2}\left(r^{2} u\right)_{\tau} d x
\end{aligned}
$$

A complicated computation gives

$$
\begin{aligned}
& \frac{d}{d \tau} \int_{0}^{+\infty}\left(r^{2} u\right)_{\tau}^{2} r^{-4} d x+\int_{0}^{+\infty} \rho^{2}\left(r^{2} u\right)_{x \tau}^{2} d x \\
& \leq C \int_{0}^{+\infty}\left(r^{2} u\right)_{\tau}^{2} r^{-4} d x+C \sup _{\tau \in[0, T]}\left\|\left(r^{2} u\right)_{x}^{2}\right\|_{L^{\infty}} \int_{0}^{+\infty}\left(r^{2} u\right)_{x}^{2} d x \\
& \quad+C \int_{0}^{+\infty}\left(\frac{u^{2}}{r^{2}}+u_{x}^{2} r^{4}\right) d x\left(1+\sup _{\tau \in[0, T]} \int_{0}^{+\infty}\left(r^{2} u\right)_{x}^{2} d x\right),
\end{aligned}
$$

integrating (3.27) with respect to $\tau$, by means of (3.3) 2 , (3.5), (3.8), (3.9), and (3.25), it holds that

$$
\begin{aligned}
& \int_{0}^{+\infty}\left(r^{2} u\right)_{\tau}^{2} r^{-4} d x+\int_{0}^{\tau} \int_{0}^{+\infty} \rho^{2}\left(r^{2} u\right)_{x s}^{2} d x d s \\
& \leq C+C \int_{0}^{\tau} \int_{0}^{+\infty}\left(r^{2} u\right)_{s}^{2} r^{-4} d x d s+C \sup _{\tau \in[0, T]}\left\|\left(r^{2} u\right)_{x}^{2}\right\|_{L^{\infty}}+C \sup _{\tau \in[0, T]} \int_{0}^{+\infty}\left(r^{2} u\right)_{x}^{2} d x \\
& \leq C+C \int_{0}^{\tau} \int_{0}^{+\infty}\left(r^{2} u\right)_{s}^{2} r^{-4} d x d s+C \sup _{\tau \in[0, T]}\left(\int_{0}^{+\infty}\left(r^{2} u\right)_{x}^{2} d x\right)^{1 / 2}\left(\int_{0}^{+\infty}\left(r^{2} u\right)_{x x}^{2} d x\right)^{1 / 2} \\
& \quad+C \sup _{\tau \in[0, T]} \int_{0}^{+\infty}\left(r^{2} u\right)_{x}^{2} d x \\
& \leq C+C e \int_{0}^{\tau} \int_{0}^{+\infty} \rho^{2}\left(r^{2} u\right)_{x s}^{2} d x d s+C e \int_{0}^{+\infty}\left(r^{2} u\right)_{\tau}^{2} r^{-4} d x
\end{aligned}
$$

choosing the constant $\epsilon$ small sufficiently, we can complete the proof of Lemma 3.3. 
Remark 3.4. By Lemmas 3.1-3.3, the following inequality holds:

$$
\begin{aligned}
\int_{0}^{+\infty} & u^{2} d x+\int_{0}^{+\infty}(\rho-\bar{\rho})^{2} d x+\int_{0}^{+\infty} u_{x}^{2} d x+\int_{0}^{+\infty} u_{\tau}^{2} d x+\int_{0}^{+\infty} \rho_{x}^{2} d x \\
& +\int_{0}^{\tau} \int_{0}^{+\infty} \rho_{x}^{2} d x d s+\int_{0}^{\tau} \int_{0}^{+\infty} u_{x}^{2} d x d s+\int_{0}^{\tau} \int_{0}^{+\infty} u_{s}^{2} d x d s \\
& +\int_{0}^{\tau} \int_{0}^{+\infty} u_{x x}^{2} d x d s+\int_{0}^{\tau} \int_{0}^{+\infty} u_{x \tau}^{2} d x d s \leq C .
\end{aligned}
$$

Lemma 3.5. Under the conditions in Theorem 2.1, it holds for any solution $(\rho, u)$ to the exterior problem (3.3) that

$$
\|(\rho-\bar{\rho}, u)(\cdot, \tau)\|_{L^{\infty}([0,+\infty))} \longrightarrow 0, \quad \tau \longrightarrow+\infty,
$$

where $C>0$ denotes a constant independent of time.

Proof. From Lemmas 3.1-3.3, we can obtain

$$
\begin{gathered}
\int_{0}^{\infty}\left\|(\rho-\bar{\rho}, u)_{x}\right\|_{L^{2}([0,+\infty))}^{2} d \tau \leq r_{-}^{-4} \int_{0}^{\infty}\left\|(\rho-\bar{\rho}, u)_{x} r^{2}\right\|_{L^{2}([0,+\infty))}^{2} d \tau \leq C, \\
\int_{0}^{\infty}\left|\frac{d}{d \tau}\left\|(\rho-\bar{\rho}, u)_{x}\right\|_{L^{2}([0,+\infty))}^{2}\right| d \tau \\
=\int_{0}^{\infty}\left|\int_{0}^{+\infty}\left(-4 \rho \rho_{x}^{2}\left(r^{2} u\right)_{x}-2 \rho^{2} \rho_{x}\left(r^{2} u\right)_{x x}\right) d x+2 \int_{0}^{+\infty} u_{x} u_{x \tau} d x\right| d \tau \\
\leq C \int_{0}^{\infty} \int_{0}^{+\infty} \rho_{x}^{2} d x d \tau+C \int_{0}^{\infty} \int_{0}^{+\infty}\left(\frac{u^{2}}{r^{2}}+u_{x}^{2} r^{4}+\left(r^{2} u\right)_{x x}^{2}+u_{x \tau}^{2}\right) d x d \tau \leq C,
\end{gathered}
$$

which together with (3.31) implies

$$
\left\|(\rho-\bar{\rho}, u)_{x}\right\|_{L^{2}([0,+\infty))}^{2} \in W^{1,1}\left(R^{+}\right) .
$$

It holds from Gagliardo-Nirenberg-Sobolev inequality that

$$
\|(\rho-\bar{\rho}, u)\|_{L^{\infty}([0,+\infty))} \leq\|(\rho-\bar{\rho}, u)\|_{L^{2}([0,+\infty))}^{1 / 2}\left\|(\rho-\bar{\rho}, u)_{x}\right\|_{L^{2}([0,+\infty))^{\prime}}^{1 / 2}
$$

which together with (3.5), (3.9), and (3.33) implies this lemma.

\subsection{Proof of Theorem 2.1}

Proof. The global existence of unique strong solution to the exterior problem as (2.4) and (2.5) can be established in terms of the short-time existence carried out as in [6], the uniform 
a-priori estimates and the analysis of regularities, which indeed follow from Lemmas 3.1-3.3. We omit the details. The large time behaviors follow from Lemma 3.5 directly. The proof of Theorem 2.1 is completed.

\section{Proof of the Initial Boundary Value Problem}

\subsection{The A-Priori Estimates}

Take the Lagrange coordinates transform

$$
x=\int_{r_{-}}^{r} \rho(r, t) r^{2} d r, \quad \tau=t .
$$

By (4.1) and the conservation of mass for $(\rho, u)$

$$
\int_{r_{-}}^{r_{+}} \rho(r, t) r^{2} d r=\int_{r_{-}}^{r_{+}} \rho_{0}(r) r^{2} d r=: 1
$$

the Lagrange coordinates transform (4.1) map $(r, t) \in\left[r_{-}, r_{+}\right] \times R^{+}$into $(x, \tau) \in[0,1] \times R^{+}$.

The relation between Lagrangian and Eulerian coordinates are satisfied as

$$
\frac{\partial x}{\partial r}=\rho r^{2}, \quad \frac{\partial x}{\partial t}=-\rho u r^{2},
$$

and the initial boundary value problem's (2.4) and (2.11) are reformulated to

$$
\begin{gathered}
\rho_{\tau}+\rho^{2}\left(r^{2} u\right)_{x}=0, \\
r^{-2} u_{\tau}+\left(\rho^{r}\right)_{x}=\left(\rho^{2}\left(r^{2} u\right)_{x}\right)_{x}-\frac{2 \rho_{x} u}{r}, \\
(\rho, u)(x, 0)=\left(\rho_{0}, u_{0}\right)(x), \quad x \in[0,1], \\
u(0, t)=u(1, t)=0, \quad \tau \in[0,+\infty),
\end{gathered}
$$

where the initial data satisfies

$$
\begin{gathered}
\rho_{0} \in L^{1}([0,1]) \cap W^{1, \infty}([0,1]), \quad \inf _{x \in[0,1]} \rho_{0}>\underline{\rho}>0 \\
\frac{1}{\sqrt{r^{2} \rho_{0}}}\left(r^{2} u_{0}\right) \in L^{2}([0,1]), \quad \sqrt{r^{2} \rho_{0}}\left(r^{2} u_{0}\right)_{x} \in L^{2}([0,1]) \\
\frac{1}{\sqrt{r^{2} \rho_{0}}}\left(r^{2} \rho\left(r^{2} \rho\left(r^{2} u_{0}\right)_{x}\right)_{x}\right) \in L^{2}([0,1]) .
\end{gathered}
$$

Then, we will establish the a-priori estimates for the solution $(\rho, u)$ to the initial boundary value problem (4.4). 
Lemma 4.1. Let $T>0$. Under the conditions in Theorem 2.2, it holds for any solution $(\rho, u)$ to the initial boundary value problem (4.4) that

$$
\begin{gathered}
\int_{0}^{1}\left(\frac{1}{2} u^{2}+\frac{1}{r-1} \rho^{\gamma-1}\right) d x+\int_{0}^{\tau} \int_{0}^{1}\left(\frac{2 u^{2}}{r^{2}}+\rho^{2} u_{x}^{2} r^{4}\right) d x d s \\
=\int_{0}^{1}\left(\frac{1}{2} u_{0}^{2}+\frac{1}{r-1} \rho_{0}^{\gamma-1}\right) d x, \quad \tau \in[0, T] .
\end{gathered}
$$

Proof. Multiplying $(4.4)_{2}$ by $r^{2} u$ and integrating the result with respect to $x$ over $[0,1]$, using $(4.4)_{1}$ and (4.5), we obtain

$$
\frac{d}{d \tau} \int_{0}^{1}\left(\frac{1}{2} u^{2}+\frac{1}{r-1} \rho^{\gamma-1}\right) d x+\int_{0}^{1} \rho^{2}\left(r^{2} u\right)_{x}^{2} d x=2 \int_{0}^{1} \rho\left(r u^{2}\right)_{x} d x
$$

and integrating (4.7) with respect to $\tau$, we obtain

$$
\int_{0}^{1}\left(\frac{1}{2} u^{2}+\frac{1}{r-1} \rho^{\gamma-1}\right) d x+\int_{0}^{\tau} \int_{0}^{1}\left(\frac{2 u^{2}}{r^{2}}+\rho^{2} u_{x}^{2} r^{4}\right) d x d s=\int_{0}^{1}\left(\frac{1}{2} u_{0}^{2}+\frac{1}{r-1} \rho_{0}^{\gamma-1}\right) d x
$$

Lemma 4.1 can be obtained.

Lemma 4.2. Let $T>0$. Under the conditions in Theorem 2.2, it holds for any solution $(\rho, u)$ to the initial boundary value problem (4.4) that

$$
\begin{gathered}
\frac{1}{2} \int_{0}^{1}\left(u+r^{2} \rho_{x}\right)^{2} d x+\frac{1}{\gamma-1} \int_{0}^{1} \rho^{\gamma-1} d x+\gamma \int_{0}^{\tau} \int_{0}^{1} \rho^{\gamma-1} \rho_{x}^{2} r^{4} d x d s \\
=\frac{1}{2} \int_{0}^{1}\left(u_{0}+r^{2} \rho_{0 x}\right)^{2} d x+\frac{1}{r-1} \int_{0}^{1} \rho_{0}^{\gamma-1} d x, \quad \tau \in[0, T],
\end{gathered}
$$

where $C$ is a positive constant independent of time.

Proof. Differentiating (4.4) $)_{1}$ with respect to $x$, we have

$$
\rho_{x \tau}+\left(\rho^{2}\left(r^{2} u\right)_{x}\right)_{x}=0,
$$

which together with $(4.4)_{2}$ and $\partial r / \partial \tau=u$ gives

$$
\left(r^{-2} u+\rho_{x}\right)_{\tau}+\left(\rho^{r}\right)_{x}=-2 r^{-3} u^{2}-\frac{2 \rho_{x} u}{r}
$$


Multiplying (4.11) by $\left(u+r^{2} \rho_{x}\right) r^{2}$, and integrating the result with respect to $x$ and $\tau$, it holds that

$$
\begin{aligned}
& \frac{1}{2} \int_{0}^{1}\left(u+r^{2} \rho_{x}\right)^{2} d x+\frac{1}{\gamma-1} \int_{0}^{1} \rho^{\gamma-1} d x+\gamma \int_{0}^{\tau} \int_{0}^{1} \rho^{\gamma-1} \rho_{x}^{2} r^{4} d x d s \\
& \quad=\frac{1}{2} \int_{0}^{1}\left(u_{0}+r^{2} \rho_{0 x}\right)^{2} d x+\frac{1}{r-1} \int_{0}^{1} \rho_{0}^{\gamma-1} d x .
\end{aligned}
$$

The proof of (4.9) is completed.

Lemma 4.3. Let $T>0$. Under the conditions in Theorem 2.2, there exists a constant $\rho^{*}>0$ such that

$$
0<\rho(x, \tau) \leq \rho^{*}, \quad(x, \tau) \in[0,1] \times[0, T] .
$$

Proof. It follows from (4.6) and (4.9) that

$$
\begin{aligned}
\rho(x, \tau) & =\rho(r, t) \leq \int_{r_{-}}^{r_{+}} \rho(r, t) d r+\int_{r_{-}}^{r_{+}}\left|\rho_{r}(r, t)\right| d r \\
& \leq r_{-}^{-2} \int_{r_{-}}^{r_{+}} \rho(r, t) r^{2} d r+r_{-}^{-2} \int_{r_{-}}^{r_{+}} \sqrt{\rho} \frac{\left|\rho_{r}(r, t)\right|}{\sqrt{\rho}} r^{2} d r \\
& \leq C+C \int_{r_{-}}^{r_{+}} \frac{1}{\rho}\left|\rho_{r}(r, t)\right|^{2} r^{2} d r \\
& \leq C+C \int_{0}^{1}\left|\rho_{x}(x, \tau)\right|^{2} r^{4} d x \leq C=: \rho^{*} .
\end{aligned}
$$

Lemma 4.4. Let $T>0$. Under the conditions in Theorem 2.2, it holds for any solution $(\rho, u)$ to the initial boundary value problem (4.4) that

$$
\int_{0}^{1} u^{2 n} d x+\int_{0}^{\tau} \int_{0}^{1}\left(\frac{u^{2 n}}{r^{2}}+\rho^{2} u^{2 n-2} u_{x}^{2} r^{4}\right) d x d s \leq C(T)
$$

for any positive integer $n \in N$, where $C(T)$ is a positive constant dependent of time.

Proof. Multiplying $(4.4)_{2}$ with $u^{2 n-1}$, integrating by parts over $[0,1]$, we have

$$
\frac{1}{2 n} \frac{d}{d \tau} \int_{0}^{1} u^{2 n} d x+\int_{0}^{1} \rho^{2}\left(r^{2} u\right)_{x}\left(r^{2} u^{2 n-1}\right)_{x} d x=\int_{0}^{1}\left(\rho^{\gamma}\left(r^{2} u^{2 n-1}\right)_{x}+\rho\left(2 r u^{2 n}\right)_{x}\right) d x .
$$


Since it holds that

$$
\begin{aligned}
\left(r^{2} u\right)_{x}\left(r^{2} u^{2 n-1}\right)_{x} & =\left(\frac{2 u}{\rho r}+r^{2} u_{x}\right)\left(\frac{2 u^{2 n-1}}{\rho r}+(2 n-1) r^{2} u^{2 n-2} u_{x}\right) \\
& =\frac{4 u^{2 n}}{\rho^{2} r^{2}}+(2 n-1) u^{2 n-2} u_{x}^{2} r^{4}+\frac{4 n u^{2 n-1} u_{x} r}{\rho},
\end{aligned}
$$

it follows from (4.16) that

$$
\begin{aligned}
& \frac{d}{d \tau} \int_{0}^{1} \frac{u^{2 n}}{2 n} d x+2 \int_{0}^{1} \frac{u^{2 n}}{r^{2}} d x+(2 n-1) \int_{0}^{1} \rho^{2} u^{2 n-2} u_{x}^{2} r^{4} d x \\
& \quad=2 \int_{0}^{1} \frac{\rho^{\gamma-1} u^{2 n-1}}{r} d x+(2 n-1) \int_{0}^{1} \rho^{r} u^{2 n-2} u_{x} r^{2} d x \\
& \quad \leq \int_{0}^{1} \frac{u^{2 n}}{r^{2}} d x+\int_{0}^{1} \rho^{2} u^{2 n-2} u_{x}^{2} r^{4} d x+C\|\rho\|_{L^{\infty}}^{2(\gamma-1)} \int_{0}^{1} u^{2 n-2} d x
\end{aligned}
$$

which together with (4.13) and Young's inequality yields

$$
\frac{d}{d \tau} \int_{0}^{1} u^{2 n} d x+\int_{0}^{1} \frac{u^{2 n}}{r^{2}} d x+\int_{0}^{1} \rho^{2} u^{2 n-2} u_{x}^{2} r^{4} d x \leq C+\int_{0}^{1} u^{2 n} d x
$$

and by applying the Gronwall's inequality to (4.19), we can obtain (4.15).

Lemma 4.5. Let $T>0$, for $n \in N$, and $n>1 / 2(\gamma-1)$. Under the conditions in Theorem 2.2, it holds for any solution $(\rho, u)$ to the initial boundary value problem (4.4) that

$$
\begin{gathered}
\int_{0}^{\tau}\left\|\rho^{2 n(\gamma-1)} u^{2 n}\right\|_{L^{\infty}([0,1])} d s \leq C(T), \\
\int_{0}^{\tau}\left\|\left|\left(\rho^{\gamma}\right)_{x} r^{2}\right|^{2 n}\right\|_{L^{\infty}([0,1])} d s \leq C(T), \quad \tau \in[0, T],
\end{gathered}
$$

where $C(T)$ is a positive constant dependent of time. 
Proof. By means of Sobolev imbedding theorem and Cauchy-Schwarz inequality, applying (4.6), (4.13), and (4.15), we get

$$
\begin{aligned}
& \int_{0}^{\tau}\left\|\rho^{2 n(\gamma-1)} u^{2 n}\right\|_{L^{\infty}([0,1])} d s \\
& \leq \int_{0}^{\tau} \int_{0}^{1}\left|\rho^{2 n(\gamma-1)} u^{2 n}\right| d x d s+\int_{0}^{\tau} \int_{0}^{1}\left|\left(\rho^{2 n(\gamma-1)} u^{2 n}\right)_{x}\right| d x d s \\
& \leq C(T)+C \int_{0}^{\tau} \int_{0}^{1} \rho^{2 n(\gamma-1)-1}\left|\rho_{x}\right| u^{2 n} d x d s+C \int_{0}^{\tau} \int_{0}^{1} \rho^{2 n(\gamma-1)}\left|u^{2 n-1} u_{x}\right| d x d s \\
& \leq C(T)+C \int_{0}^{\tau} \int_{0}^{1}\left|\rho_{x}\right|^{2} r^{4} d x d s+C \int_{0}^{\tau} \int_{0}^{1} \rho^{2(2 n(\gamma-1)-1)} u^{4 n} r^{-4} d x d s \\
& \quad+\int_{0}^{\tau} \int_{0}^{1} \rho^{2(2 n(\gamma-1)-1)} u^{2 n} r^{-4} d x d s+C \int_{0}^{\tau} \int_{0}^{1} \rho^{2} u^{2 n-2} u_{x}^{2} r^{4} d x d s \leq C(T) .
\end{aligned}
$$

Next, we find that

$$
\rho_{x} r^{2}=\rho_{0 x} r_{0}^{2}+u_{0}-u-\int_{0}^{\tau}\left(\rho^{r}\right)_{x} r^{2} d s
$$

which together with (4.13), (4.15), and (4.22) gives

$$
\begin{aligned}
& \int_{0}^{\tau}\left\|\left|\left(\rho^{r}\right)_{x} r^{2}\right|^{2 n}\right\|_{L^{\infty}} d s \\
& \quad=r^{2 n} \int_{0}^{\tau}\left\|\rho^{2 n(\gamma-1)}\left|\rho_{x} r^{2}\right|^{2 n}\right\|_{L^{\infty}} d s \\
& \quad=r^{2 n} \int_{0}^{\tau}\left\|\rho^{2 n(\gamma-1)}\left|\rho_{0 x} r_{0}^{2}+u_{0}-u-\int_{0}^{s}\left(\rho^{r}\right)_{x} r^{2} d l\right|^{2 n}\right\|_{L^{\infty}} d s \\
& \quad \leq C \int_{0}^{\tau}\left\|\rho^{2 n(\gamma-1)}\left(\rho_{0 x}^{2 n}+u_{0}^{2 n}+u^{2 n}\right)\right\|_{L^{\infty}} d s+C \int_{0}^{\tau}\left\|\rho^{2 n(\gamma-1)}\left(\int_{0}^{s}\left(\rho^{r}\right)_{x} r^{2} d l\right)^{2 n}\right\|_{L^{\infty}} d s \\
& \quad \leq C(T)+C \int_{0}^{\tau}\left\|\rho^{2 n(r-1)} u^{2 n}\right\|_{L^{\infty}} d s+C(T) \int_{0}^{\tau} \int_{0}^{s}\left\|\left|\left(\rho^{r}\right)_{x} r^{2}\right|^{2 n}\right\|_{L^{\infty}} d l d s,
\end{aligned}
$$

applying the Gronwall's inequality to (4.24), we obtain (4.21).

Lemma 4.6. Let $T>0$. Under the conditions in Theorem 2.2, there exists a constant $\rho_{*}>0$ such that

$$
\rho(x, \tau) \geq \rho_{*}>0, \quad(x, \tau) \in[0,1] \times[0, T] .
$$


Proof. It is easy to verify that

$$
\begin{aligned}
\overline{\rho^{(\gamma+1) / 2}}(\tau) & =: \int_{0}^{1} \rho^{(\gamma+1) / 2}(x, \tau) d x \geq \frac{r_{-}^{2}}{r_{+}^{2}}\left(\int_{r_{-}}^{r_{+}} \rho(r, t) r^{2} d r\right)^{1+(\gamma+1) / 2} \\
& =\frac{r_{-}^{2}}{r_{+}^{2}}\left(\int_{r_{-}}^{r_{+}} \rho_{0}(r) r^{2} d r\right)^{1+(\gamma+1) / 2}>0 \\
& \left\|\rho^{(\gamma+1) / 2}-\overline{\rho^{(\gamma+1) / 2}}(\tau)\right\|_{L^{2}([0,1])}^{2} \in W^{1,1}([0, T])
\end{aligned}
$$

Indeed, it holds that

$$
\int_{0}^{\tau}\left\|\rho^{(\gamma+1) / 2}-\overline{\rho^{(\gamma+1) / 2}}(s)\right\|_{L^{2}([0,1])}^{2} d s \leq C \int_{0}^{\tau}\left\|\left(\rho^{(\gamma+1) / 2}\right)_{x} r^{2}\right\|_{L^{2}([0,1])}^{2} d s \leq C,
$$

which together with (4.4), (4.9), and (4.13) gives

$$
\begin{aligned}
& \int_{0}^{\tau}\left|\frac{d}{d s}\left\|\rho^{(\gamma+1) / 2}-\overline{\rho^{(\gamma+1) / 2}}(s)\right\|_{L^{2}([0,1])}^{2}\right| d s \\
& \quad \leq(\gamma+1) \int_{0}^{\tau}\left|\int_{0}^{1}\left(\rho^{(\gamma+1) / 2}-\overline{\rho^{(\gamma+1) / 2}}(s)\right) \rho^{(\gamma+1) / 2-1} \rho_{s} d x\right| d s \\
& \quad+2 \int_{0}^{\tau}\left|\int_{0}^{1}\left(\rho^{(\gamma+1) / 2}-\overline{\rho^{(\gamma+1) / 2}}(s)\right)\left(\overline{\rho^{(\gamma+1) / 2}}(s)\right)_{s} d x\right| d s \\
& \leq C \int_{0}^{\tau} \int_{0}^{1}\left(\rho^{(\gamma+1) / 2}-\overline{\rho^{(\gamma+1) / 2}}(s)\right)^{2} d x d s+\int_{0}^{\tau} \int_{0}^{1} \rho^{2} u_{x}^{2} r^{4} d x d s \leq C .
\end{aligned}
$$

By Gagliardo-Nirenberg-Sobolev inequality and (4.27), it follows that

$$
\left\|\rho^{(\gamma+1) / 2}-\overline{\rho^{(\gamma+1) / 2}}(\tau)\right\|_{L^{\infty}([0,1])}^{2} \longrightarrow 0 \quad \text { as } \tau \longrightarrow+\infty
$$

Thus, there is a $T_{0}>0$ and a constant $\rho_{1}>0$ such that

$$
\rho(x, \tau) \geq \rho_{1}, \quad x \in[0,1], \tau \in\left[T_{0},+\infty\right) .
$$

For $\tau \in\left[0, T_{0}\right]$, denote

$$
v(x, \tau)=: \frac{1}{\rho(x, \tau) r^{2}(x, \tau)}
$$


then from (4.4), we can obtain

$$
v_{\tau}=\frac{\left(r^{2} u\right)_{x}}{r^{2}}-\frac{2 v u}{r}
$$

multiplying (4.33) by $4 v^{3}$ and integrating the result over $[0,1] \times\left[0, T_{0}\right]$, we have

$$
\begin{aligned}
\int_{0}^{1} v^{4} d x= & \int_{0}^{1} v_{0}^{4} d x+4 \int_{0}^{\tau} \int_{0}^{1} v^{3} \frac{\left(r^{2} u\right)_{x}}{r^{2}} d x d s-8 \int_{0}^{\tau} \int_{0}^{1} \frac{v^{4} u}{r} d x d s \\
= & \int_{0}^{1} v_{0}^{4} d x+12 \int_{0}^{\tau} \int_{0}^{1} v^{4} u\left(\rho_{x} r^{2}\right) d x d s+24 \int_{0}^{\tau} \int_{0}^{1} v^{4} \frac{u}{r} d x d s \\
= & \int_{0}^{1} v_{0}^{4} d x+12 \int_{0}^{\tau} \int_{0}^{1} v^{4} u\left(\rho_{0 x} r_{0}^{2}+u_{0}-u-\int_{0}^{s}\left(\rho^{r}\right)_{x} r^{2} d l\right) d x d s \\
& +24 \int_{0}^{\tau} \int_{0}^{1} v^{4} \frac{u}{r} d x d s .
\end{aligned}
$$

From (4.21), it holds that

$$
\begin{aligned}
& \int_{0}^{1} v^{4} d x+12 \int_{0}^{\tau} \int_{0}^{1} v^{4} u^{2} d x d s \\
& \quad=\int_{0}^{1} v_{0}^{4} d x+12 \int_{0}^{\tau} \int_{0}^{1} v^{4} u \rho_{0 x} r_{0}^{2} d x d s+12 \int_{0}^{\tau} \int_{0}^{1} v^{4} u u_{0} d x d s \\
& \quad-12 \int_{0}^{\tau} \int_{0}^{1} v^{4} u\left(\int_{0}^{s}\left(\rho^{r}\right)_{x} r^{2} d l\right) d x d s+24 \int_{0}^{\tau} \int_{0}^{1} v^{4} \frac{u}{r} d x d s \\
& \quad \leq 6 \int_{0}^{\tau} \int_{0}^{1} v^{4} u^{2} d x d s+C\left(T_{0}\right) \int_{0}^{\tau} \int_{0}^{1} v^{4} d x d s,
\end{aligned}
$$

which yields to

$$
\int_{0}^{1} v^{4} d x \leq C+C\left(T_{0}\right) \int_{0}^{\tau} \int_{0}^{1} v^{4} d x d s
$$

where $C\left(T_{0}\right)$ is a positive constant dependent of time $T_{0}$. By Gronwall's inequality, (4.36) leads to

$$
\int_{0}^{1} v^{4} d x=\int_{0}^{1} \frac{1}{\rho^{4} r^{8}} d x \leq C\left(T_{0}\right)
$$


Journal of Applied Mathematics

19

It holds for $(x, \tau) \in([0,1]) \times\left[0, T_{0}\right]$ that

$$
\begin{aligned}
\frac{1}{\rho} & =\int_{0}^{1} \frac{1}{\rho} d x+\int_{0}^{1}\left|\left(\frac{1}{\rho}\right)_{x}\right| d x \\
& \leq C+C \int_{0}^{1} \frac{1}{\rho^{4} r^{8}} d x+C\left(\int_{0}^{1} \frac{1}{\rho^{4} r^{8}} d x\right)^{1 / 2}\left(\int_{0}^{1} \rho_{x}^{2} r^{4} d x\right)^{1 / 2} \leq C\left(T_{0}\right)
\end{aligned}
$$

namely,

$$
\rho(x, \tau) \geq C\left(T_{0}\right)=: \rho_{2}, \quad(x, \tau) \in([0,1]) \times\left[0, T_{0}\right] .
$$

Therefore, we can choose

$$
\rho_{*}=\min \left\{\rho_{1}, \rho_{2}\right\},
$$

to get

$$
\rho \geq \rho_{*,} \quad(x, \tau) \in([0,1]) \times[0,+\infty) .
$$

Lemma 4.7. Let $T>0$. Under the conditions in Theorem 2.2, it holds for any solution $(\rho, u)$ to the initial boundary value problem (4.4) that

$$
\begin{aligned}
& \int_{0}^{1}\left(r^{2} u\right)_{x}^{2} d x+\int_{0}^{1}\left(r^{2} u\right)_{\tau}^{2} r^{-4} d x+\int_{0}^{\tau} \int_{0}^{1}\left(r^{2} u\right)_{s}^{2} r^{-4} d x d s \\
& \quad+\int_{0}^{\tau} \int_{0}^{1} \rho^{2}\left(r^{2} u\right)_{x s}^{2} d x d s+\int_{0}^{\tau} \int_{0}^{1}\left(r^{2} u\right)_{x x}^{2} d x d s \leq C, \quad \tau \in[0, T],
\end{aligned}
$$

where $C>0$ denotes a constant independent of time.

Proof. The proof is similar to Lemma 3.3. We omit here.

Remark 4.8. By Lemmas 4.1-4.7, the following inequality holds:

$$
\begin{gathered}
\int_{0}^{1} u^{2} d x+\int_{0}^{1}(\rho-\bar{\rho})^{2} d x+\int_{0}^{1} u_{x}^{2} d x+\int_{0}^{1} u_{\tau}^{2} d x+\int_{0}^{1} \rho_{x}^{2} d x \\
+\int_{0}^{\tau} \int_{0}^{1} \rho_{x}^{2} d x d s+\int_{0}^{\tau} \int_{0}^{1} u_{x}^{2} d x d s+\int_{0}^{\tau} \int_{0}^{1} u_{s}^{2} d x d s \\
+\int_{0}^{\tau} \int_{0}^{1} u_{x x}^{2} d x d s+\int_{0}^{\tau} \int_{0}^{1} u_{x \tau}^{2} d x d s \leq C .
\end{gathered}
$$


Lemma 4.9. Under the conditions in Theorem 2.2, it holds for any solution $(\rho, u)$ to the initial boundary value problems (2.4) and (2.11) that

$$
\|(\rho-\tilde{\rho}, u)(\cdot, t)\|_{L^{\infty}\left(\left[r_{-}, r_{+}\right]\right)} \leq C_{0} e^{-C_{1} t}, \quad t>0,
$$

where $C_{0}$ and $C_{1}$ denote the constants independent of time and $\tilde{\rho}=\int_{r_{-}}^{r_{+}} \rho(r, t) r^{2} d r$.

Proof. In a similar argument to show (4.8) and (4.12) with modification, we can obtain the following

$$
\begin{gathered}
\frac{d}{d t} \int_{r_{-}}^{r_{+}}\left(\frac{1}{2} \rho u^{2}+\frac{1}{r-1}\left(\rho^{r}-\tilde{\rho}^{r}-\gamma \tilde{\rho}^{r-1}(\rho-\tilde{\rho})\right)\right) r^{2} d r+\int_{r_{-}}^{r_{+}}\left(2 \rho u^{2}+\rho u_{r}^{2} r^{2}\right) d r=0, \\
\frac{1}{2} \frac{d}{d t} \int_{r_{-}}^{r_{+}} \rho\left(u+\rho^{-1} \rho_{r}\right)^{2} r^{2} d r+\frac{1}{\gamma-1} \frac{d}{d t} \int_{r_{-}}^{r_{+}}\left(\rho^{r}-\tilde{\rho}^{\gamma}-\gamma \tilde{\rho}^{\gamma-1}(\rho-\tilde{\rho})\right) r^{2} d r \\
+\gamma \int_{r_{-}}^{r_{+}} \rho^{\gamma-2} \rho_{r}^{2} r^{2} d r=0 .
\end{gathered}
$$

It holds from (4.9), (4.13), and (4.25) that

$$
\int_{r_{-}}^{r_{+}}(\rho-\tilde{\rho})^{2} r^{2} d r \leq C \int_{r_{-}}^{r_{+}} \rho^{r-2} \rho_{r}^{2} r^{2} d r
$$

Denote

$$
E(t)=: \int_{r_{-}}^{r_{+}} \rho\left(u^{2}+\left(u+\rho^{-1} \rho_{r}\right)^{2}\right) r^{2} d r+\int_{r_{-}}^{r_{+}}\left(\rho^{r}-\tilde{\rho}^{r}-\gamma \widetilde{\rho}^{r-1}(\rho-\widetilde{\rho})\right) r^{2} d r .
$$

By (4.45)-(4.48), a complicated computation gives rise to

$$
\frac{d}{d t} E(t)+\bar{C} E(t) \leq 0
$$

which gives

$$
E(t) \leq E(0) e^{-\bar{C} t}
$$

By the fact that

$$
E(t) \geq c\left(\|u\|_{L^{2}\left(\left[r_{-}, r_{+}\right]\right)}^{2}+\|\rho-\tilde{\rho}\|_{L^{2}\left(\left[r_{-}, r_{+}\right]\right)}^{2}+\left\|\rho_{r}\right\|_{L^{2}\left(\left[r_{-}, r_{+}\right]\right)}^{2}\right),
$$


where $c>0$ is a constant independent of time and Gagliardo-Nirenberg-Sobolev inequality

$$
\|(\rho-\tilde{\rho}, u)\|_{L^{\infty}\left(\left[r_{-}, r_{+}\right]\right)} \leq C\|(\rho-\tilde{\rho}, u)\|_{L^{2}\left(\left[r_{-}, r_{+}\right]\right)}^{1 / 2}\left\|(\rho-\tilde{\rho}, u)_{r}\right\|_{L^{2}\left(\left[r_{-}, r_{+}\right]\right)^{\prime}}^{1 / 2}
$$

we obtain (4.44).

\subsection{Proof of Theorem 2.2}

Proof. The proof of Theorem 2.2 is similar to Theorem 2.1. We omit the details.

\section{Acknowledgments}

The research of R. Lian is partially supported by NNSFC no. 11101145. The research of L. Huang is partially supported by NNSFC no. 11126323.

\section{References}

[1] D. Bresch and B. Desjardins, "Existence of global weak solutions for a 2D viscous shallow water equations and convergence to the quasi-geostrophic model," Communications in Mathematical Physics, vol. 238, no. 1-2, pp. 211-223, 2003.

[2] D. Bresch and B. Desjardins, "On the construction of approximate solutions for the 2D viscous shallow water model and for compressible Navier-Stokes models," Journal de Mathématiques Pures et Appliquées Neuvième Série, vol. 86, no. 4, pp. 362-368, 2006.

[3] J.-F. Gerbeau and B. Perthame, "Derivation of viscous Saint-Venant system for laminar shallow water; numerical validation," Discrete and Continuous Dynamical Systems Series B, vol. 1, no. 1, pp. 89-102, 2001.

[4] F. Marche, "Derivation of a new two-dimensional viscous shallow water model with varying topography, bottom friction and capillary effects," European Journal of Mechanics B, vol. 26, no. 1, pp. 49-63, 2007.

[5] Z. Guo, Q. Jiu, and Z. Xin, "Spherically symmetric isentropic compressible flows with densitydependent viscosity coefficients," SIAM Journal on Mathematical Analysis, vol. 39, no. 5, pp. 1402-1427, 2008.

[6] S. Jiang, Z. Xin, and P. Zhang, "Global weak solutions to 1D compressible isentropic Navier-Stokes equations with density-dependent viscosity," Methods and Applications of Analysis, vol. 12, no. 3, pp. 239-251, 2005.

[7] T.-P. Liu, Z. Xin, and T. Yang, "Vacuum states for compressible flow," Discrete and Continuous Dynamical Systems, vol. 4, no. 1, pp. 1-32, 1998.

[8] M. Okada, Š. M. Nečasová, and T. Makino, "Free boundary problem for the equation of onedimensional motion of compressible gas with density-dependent viscosity," Annali dell'Università di Ferrara Nuova Serie, vol. 48, pp. 1-20, 2002.

[9] S.-W. Vong, T. Yang, and C. Zhu, "Compressible Navier-Stokes equations with degenerate viscosity coefficient and vacuum. II," Journal of Differential Equations, vol. 192, no. 2, pp. 475-501, 2003.

[10] T. Yang, Z. Yao, and C. Zhu, "Compressible Navier-Stokes equations with density-dependent viscosity and vacuum," Communications in Partial Differential Equations, vol. 26, no. 5-6, pp. 965-981, 2001.

[11] T. Yang and H. Zhao, "A vacuum problem for the one-dimensional compressible Navier-Stokes equations with density-dependent viscosity," Journal of Differential Equations, vol. 184, no. 1, pp. 163$184,2002$.

[12] T. Yang and C. Zhu, "Compressible Navier-Stokes equations with degenerate viscosity coefficient and vacuum," Communications in Mathematical Physics, vol. 230, no. 2, pp. 329-363, 2002. 
[13] A. Mellet and A. Vasseur, "Existence and uniqueness of global strong solutions for one-dimensional compressible Navier-Stokes equations," SIAM Journal on Mathematical Analysis, vol. 39, no. 4, pp. 1344-1365, 2007/08.

[14] Q. Jiu and Z. Xin, "The Cauchy problem for 1D compressible flows with density-dependent viscosity coefficients," Kinetic and Related Models, vol. 1, no. 2, pp. 313-330, 2008.

[15] Q. Jiu, Y. Wang, and Z. Xin, "Stability of rarefaction waves to the 1D compressible Navier-Stokes equations with density-dependent viscosity," Communications in Partial Differential Equations, vol. 36, no. 4, pp. 602-634, 2011.

[16] H.-L. Li, J. Li, and Z. Xin, "Vanishing of vacuum states and blow-up phenomena of the compressible Navier-Stokes equations," Communications in Mathematical Physics, vol. 281, no. 2, pp. 401-444, 2008.

[17] R. Lian, Z. Guo, and H.-L. Li, “Dynamical behaviors for 1D compressible Navier-Stokes equations with density-dependent viscosity," Journal of Differential Equations, vol. 248, no. 8, pp. 1926-1954, 2010. 


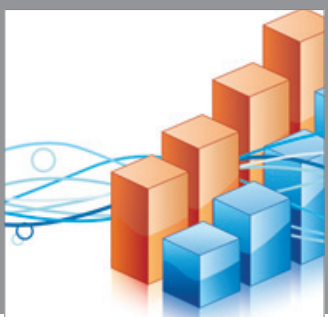

Advances in

Operations Research

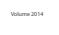

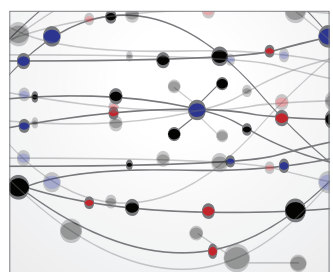

\section{The Scientific} World Journal
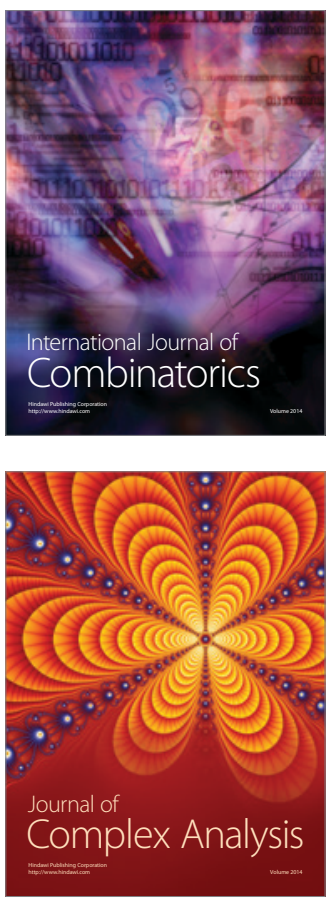

International Journal of

Mathematics and

Mathematical

Sciences
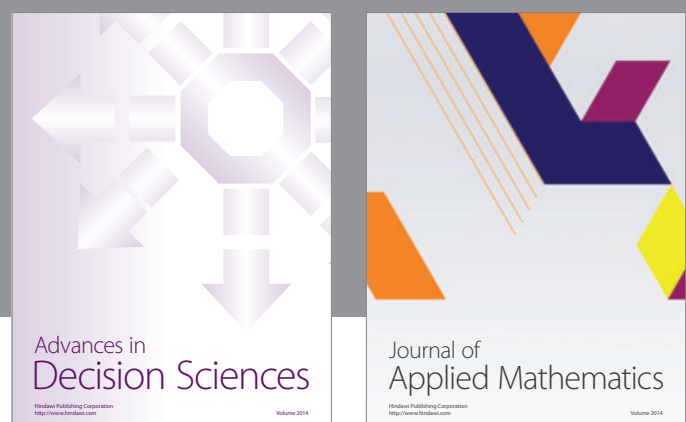

Journal of

Applied Mathematics
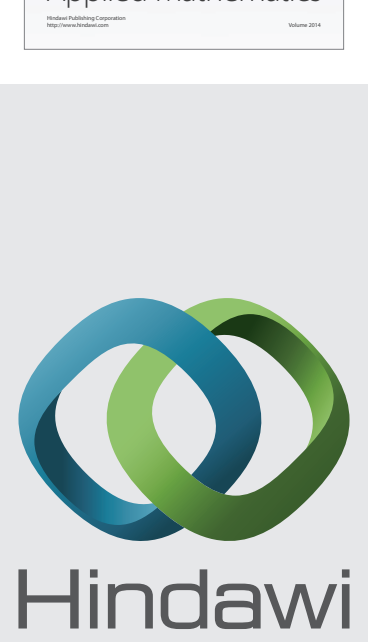

Submit your manuscripts at http://www.hindawi.com
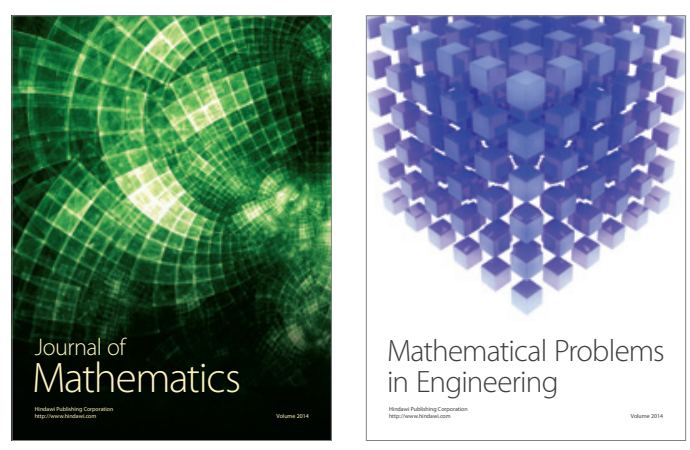

Mathematical Problems in Engineering
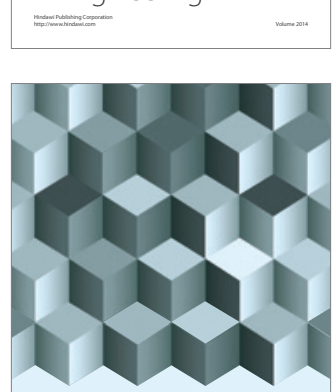

Journal of

Function Spaces
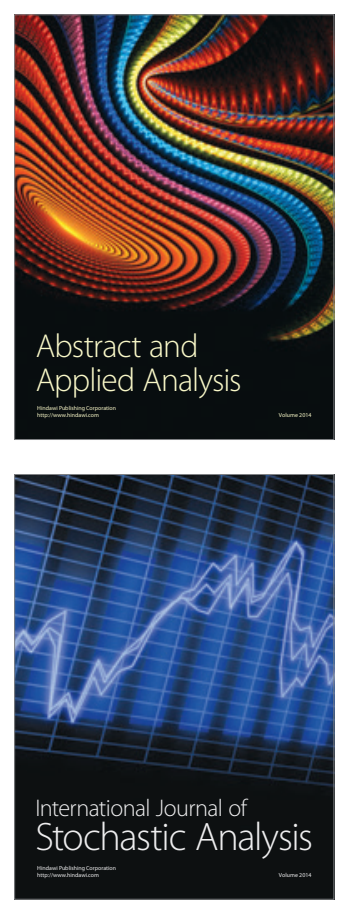

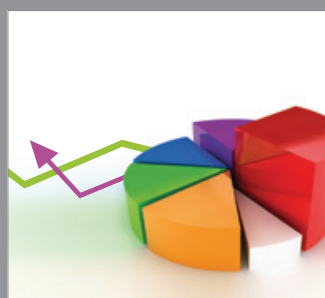

ournal of

Probability and Statistics

Promensencen
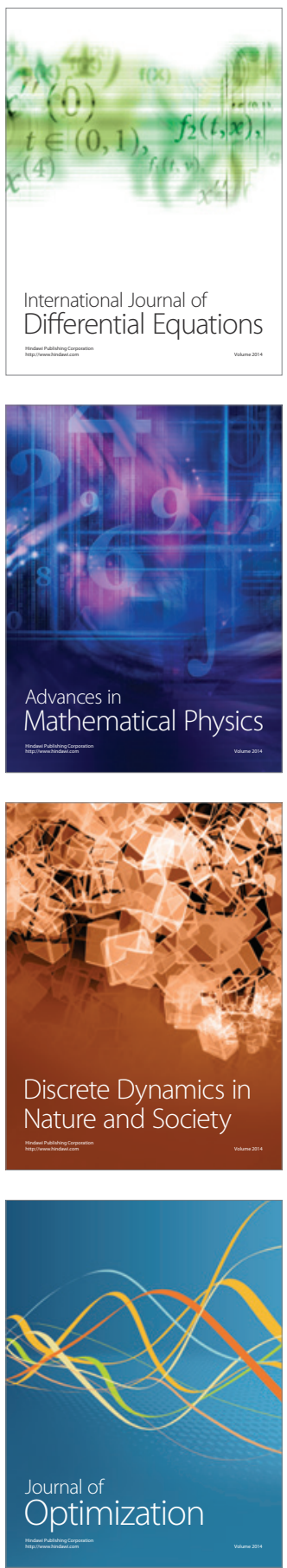